\title{
Effective contact model for transport through weakly-doped graphene
}

\author{
Henning Schomerus \\ Department of Physics, Lancaster University, Lancaster, LA1 $4 Y B$, UK
}

(Dated: September 8, 2018)

\begin{abstract}
Recent investigations address transport through ballistic charge-neutral graphene strips coupled to doped graphitic leads. This paper shows that identical transport properties arise when the leads are replaced by quantum wires. This duality between graphitic and metallic leads originates in the selection of modes with transverse momentum close to the $\mathrm{K}$ points, and can be extended to a wide class of contact models. Among this class we identify a simple, effective contact model, which provides an efficient tool to study the transport through extended weakly-doped graphitic systems.
\end{abstract}

PACS numbers: 73.63.-b, 72.10.Bg, 73.63.Bd, 81.05.Uw

\section{INTRODUCTION}

Since the recent break-through in its fabrication, $, 1,2,3,4$ graphene, the atomically thin two-dimensional hexagonal arrangement of carbon atoms, has caught the excitement of experimentalists and theoreticians alike because it possesses unique electronic properties which originate from the two conical points of its Fermi surface, located at the $\mathrm{K}$ points of the hexagonal Brillouin zone. In a simple model, a clean graphene sheet is described by a tight-binding Hamiltonian $H=-\gamma \sum_{\langle i j\rangle} c_{i}^{\dagger} c_{j}$, in which next neighbors $\langle i j\rangle$ on the hexagonal lattice (with lattice constant $a$ ) are connected by a hopping matrix element $\gamma$. The lattice supports Bloch waves with wave numbers $k_{x}, k_{y}$ and dispersion relation $E=$ $\pm \gamma\left|1+2 e^{i 3 k_{x} a / 2} \cos \left(\sqrt{3} k_{y} a / 2\right)\right|$. At the Fermi energy $E=0$ of undoped, charge neutral graphene, the Fermi surface shrinks to two inequivalent points $\left(k_{x}, k_{y}\right)=$ $\left(\frac{2 \pi}{3 a}, \pm \frac{2}{3} \frac{\pi}{\sqrt{3} a}\right)$. In the vicinity of these points the dispersion relation can be linearized, and then becomes conical with slope $|d E / d k|=3 \gamma a / 2$. The ensuing low-energy theory is described by a Dirac Hamiltonian.$^{5}$

By varying the Fermi energy via a gate voltage across the charge-neutrality point, graphene offers the unique possibility to switch the doping of the system from $\mathrm{n}$ to $\mathrm{p}$, where the doped charge density can be changed continuously. The first graphene-based field-effect transistor was demonstrated in Ref. 1. The mobility of carefully fabricated graphene flakes already exceeds 50,000 $\mathrm{cm}^{2} / \mathrm{Vs} .4$ Motivated by the ensuing prospect of graphitic electronic devices with properties very much different from semiconductor-based technology, a number of recent works have explored the phase-coherent transport properties of finite segments of graphene, connected via leads to electronic reservoirs. This resulted in the discovery of a modified quantum Hall effect ${ }^{2,3}$ with a half-integer sequence of Hall conductance plateaus. It was furthermore predicted that weak-localization corrections to the conductance depend sensitively on symmetries preserved or broken by the disorder ${ }^{6.7}$ Clean graphitic samples exhibit a finite conductivity of the order of the conductance quantum $1,2,3,8,9,10$ and a shot noise identical to a disordered quantum wire $\stackrel{8}{-}$ Transport across $p-n$ junctions was theoretically studied in Ref. 11. A similar but modified set of unique transport phenomena is found for graphene bilayers, which furthermore offer the possibility to open up a controllable gap by an electric field effect (see Ref. 12 and references therein).

A problem untouched by the recent transport investigations is the role of the leads connecting the graphitic sample to the electronic reservoirs. Some of the recent theoretical transport studies model the leads as strips of doped graphene. This type of lead supports distinctively different sets of modes than a conventional quantum wire - for the same transverse mode profile, propagating modes in a conventional quantum wire often correspond to evanescent modes in a graphene strip, and vice versa. Therefore, it could be argued that a detailed modelling of the leads is critical for the understanding of transport in graphitic systems.

The objective of this paper is to show that to the contrary, transport through a sufficiently large and only weakly doped graphene sample often does not critically depend on most details of the leads. For the particular case of quantum-wire and graphitic leads, this insensitivity manifests itself in an explicit duality, which can be formulated in terms of a shift of the gate potentials that control the charge density in the leads. This duality originates in a mode selection mechanism, which dictates that the transport in extended, only weakly doped samples of graphene is dominated by the small part of the mode space in the vicinity of the conical points. Using this universal mechanism, one is naturally led to formulate an effective contact model, which is parameterized by a single complex number $\mu$. This effective model can be applied to a much larger class of leads which are amenable to the mode-selection mechanism. The main requirement is that the leads are sufficiently wide, and provide a dense set of propagating modes with transverse wave number close to the conical points (leads which do not possess any propagating modes at these transverse wave numbers exhibit a very large contact resistance, and hence do not provide a good electronic coupling).

In striking contrast to universal transport through semiconductor quantum $\operatorname{dots}^{13}$, the effective contact model provides a unified description of leads which support a different number of propagating modes (but share 
the same width). Numerical computations confirm that the effective contact model provides an efficient and reliable tool to study the transport through extended weakly-doped graphitic systems.

This paper is organized as follows. Section $\amalg$ formally poses the problem of the lead-sensitivity of transport in mesoscopic systems. In Section III, I calculate the conductance and Fano factor of a rectangular graphene sample which is connected to quantum wires or graphitic leads. The rectangular graphene sample is held at the charge neutrality point, while the charge density in the leads is controlled by gate potentials. At a finite charge density in the leads, this deliver the duality of both contact models. In Section IV] I describe the effective contact model which represents a large class of leads, encompassing quantum wires and doped graphitic strips. Section $\nabla$ describes how the complex number $\mu$ could be extracted from the conductance and shot noise, and tests the applicability of the effective contact model via numerical computations for rectangular and circular graphene samples. Conclusions are presented in [VI] The appendices contain technical details of the calculations.

\section{FORMULATION OF THE PROBLEM}

At low bias, the phase-coherent transport properties of a mesoscopic system probed in a two-terminal geometry are encoded in the scattering matrix ${ }^{13,14}$

$$
S=\left(\begin{array}{cc}
r & t^{\prime} \\
t & r^{\prime}
\end{array}\right)
$$

which contains the transmission (reflection) amplitudes $t, t^{\prime}\left(r, r^{\prime}\right)$ of charge carriers incident from the source or drain contact, respectively. Two characteristic transport properties are the Landauer conductance

$$
G=\left(2 e^{2} / h\right) \operatorname{tr} t^{\dagger} t
$$

and the shot-noise Fano factor

$$
F=1-\frac{\operatorname{tr} t^{\dagger} t t^{\dagger} t}{\operatorname{tr} t^{\dagger} t}
$$

In general, the transmission matrix $t$ is determined by matching the propagating modes in the leads to the modes in the mesoscopic system, and hence depends on the properties of both of these constituents. This paper considers the case that the mesoscopic system is an extended, only weakly doped sample of graphene with a specified geometry, and asks the questions how the transport properties change when the sample is contacted by different types of leads (keeping the width and position of the leads fixed).

\section{DUALITY OF METALLIC AND GRAPHITIC CONTACTS}

It is instructive to first explore the potential relevance and eventual insensitivity to most details of the leads by
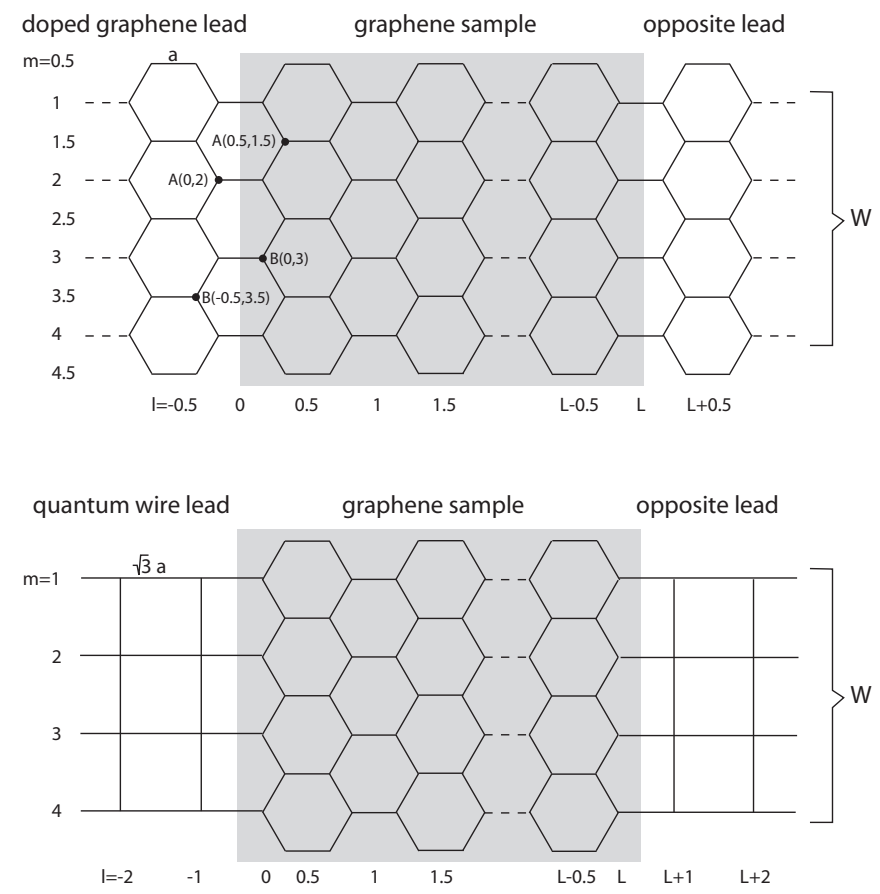

FIG. 1: A graphene sample, modelled as a hexagonal lattice (lattice constant $a$ ), is attached to leads formed either by doped graphene (top) or by a quantum wire, modelled as a commensurably matched square lattice with lattice constant $\sqrt{3} a$ (bottom). The charge carrier density in the leads is controlled by a gate potential $V_{g}$, while the central graphitic region is held close to the charge-neutrality point. The paper demonstrates that both leads are equivalent when the gate potential is suitably adjusted, and further extends this duality to a broad class of leads and contacts, which all share the properties of a simple effective contact model.

calculating the conductance for a specific example, a rectangular undoped graphene sample of width $\mathcal{W}=\sqrt{3} a W$ and length $\mathcal{L}=3 a L$, where $W, L \gg 1$ are integers (see Fig. (1). The strip is connected to leads of the same width, either formed by doped graphene or by a quantum wire, which are modelled in a tight-binding approach on a hexagonal or square lattice, respectively. The electronic density in the leads is controlled by a gate potential, inducing an on-site potential energy denoted by $V_{g}$. For $\gamma / W \ll\left|V_{g}\right| \ll \gamma$ the transport of this system connected to graphitic leads has been investigated earlier in the framework of the Dirac equation. $\frac{8}{-}$ It was found that the conductivity $\sigma=\frac{\mathcal{L}}{\mathcal{W}} G=\frac{4 e^{2}}{\pi h}$ is of order of the experimentally observed value, while the Fano factor $F=1 / 3$ coincides with the universal value of a disordered quantum wire.

A unified description of square- and hexagonal-lattice leads can be achieved when the hexagonal sublattices $A$, $B$, which differ in the orientation of the bonds, are indexed by two numbers $l, m$ which are either both integer or both half-integer (see Fig. 1). On the square lattice 
the indices $l$ and $m$ are both integer. For both types of lattice, the transverse wave function of the modes in the lead are then given by

$$
\Psi_{n m}=\sqrt{\frac{2}{W+1}} \sin \frac{n m \pi}{W+1},
$$

where $n=1,2,3, \ldots, W$ is the mode index. Associated with each transverse mode are two extended Bloch waves with longitudinal wave numbers $\pm k_{n}$, which are real for propagating modes and complex for evanescent modes. The wave numbers are fixed by the dispersion relation, which for the hexagonal lattice is given by

$$
\begin{aligned}
& V_{g}=\eta \sqrt{f_{n, k_{n}} f_{n,-k_{n}}}, \quad \eta=\operatorname{sgn} V_{g}= \pm 1, \\
& f_{n, k_{n}}=\gamma+2 \gamma e^{i 3 k_{n} a / 2} \cos \frac{n \pi}{2(W+1)},
\end{aligned}
$$

while for the square lattice

$$
V_{g}=2 \gamma \cos \left(\sqrt{3} a k_{n}\right)+2 \gamma \cos \frac{n \pi}{W+1} .
$$

Because of the different dispersion relations, the sets of transverse-mode indices $n$ supporting propagating modes on the two types of lattice are not the same. Hence, for ordinary mesoscopic systems (such as a semiconducting quantum dot, a quantum wire, a metallic nanoparticle or a hybrid structure with superconducting or ferromagnetic properties) one would obtain distinctively different transport properties when quantum wire leads would be replaced by graphitic leads.

In order to obtain the transmission amplitudes $t_{n^{\prime} n}$ when these leads are coupled to the undoped rectangular graphene sample, the modes in the leads have to be matched to modes in the graphene strip, which follow from Eq. (5) by setting $V_{g}=0$. For the present quasi-one dimensional geometry, the mode index $n$ is conserved, $\underline{8}$ and a straightforward calculation (see Appendix A) delivers

$$
t_{n^{\prime} n}=\delta_{n^{\prime} n} \frac{\mu_{n,+}-\mu_{n,-}}{\mu_{n,+} e^{-\kappa_{n} \mathcal{L}}-\mu_{n,-} e^{\kappa_{n} \mathcal{L}}},
$$

where the moduli of

$$
\kappa_{n}=\frac{2}{3 a} \ln \left(2 \cos \frac{n \pi}{2(W+1)}\right)
$$

denote the decay constants of the modes in undoped graphene. The lead dependence of the transmission coefficients is encoded in the self energies $\mu_{n, \pm}$ of the in- and outgoing modes, given by

$$
\begin{aligned}
& \mu_{n, \pm}^{(h)}=-\eta \gamma \frac{f_{n, \pm \eta k_{n}}}{\sqrt{f_{n, k_{n}} f_{n,-k_{n}}}} \quad \text { (hexagonal lattice), } \\
& \mu_{n, \pm}^{(s)}=-\gamma e^{ \pm i \sqrt{3} k_{n} a} \quad \text { (square lattice). }
\end{aligned}
$$

The effective contact model developed in Section IV arises from the observation that the detailed lead dependence embodied in these numbers becomes irrelevant

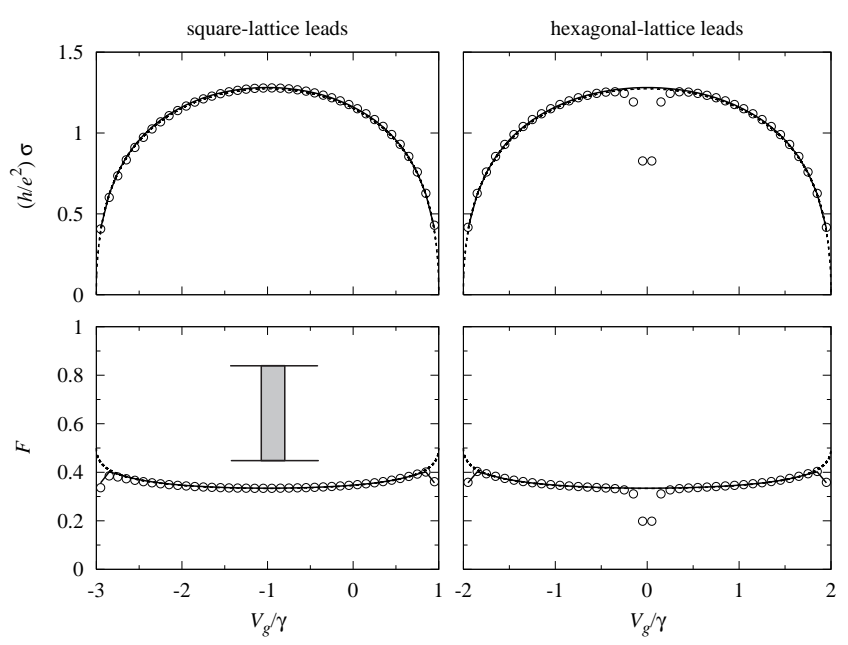

FIG. 2: Gate-voltage dependence of the conductivity $\sigma=$ $(\mathcal{L} / \mathcal{W}) G$ (in units of $\left.e^{2} / h\right)$ and the shot-noise Fano factor $F$ of an undoped graphene strip of width $\mathcal{W}=152 \sqrt{3} a$ and length $\mathcal{L}=30 \mathrm{a}$. The open data points are obtained by numerical computations for square-lattice leads (left) and hexagonallattice leads (right). The solid curves are the prediction of the effective contact model (18) with $\mu$ given by Eq. (10) or Eq. (11), respectively. The dashed curves are the analytical expressions (1213/14/15) obtained within the saddle-point approximation.

when the leads are coupled to a sufficiently large graphitic region. According to Eq. (8), all modes in this region decay rapidly with the exception of modes with index $n \approx 2(W+1) / 3 \equiv \tilde{n}$, which have transverse wave numbers in the vicinity of the conical points. For a sufficiently large sample we hence only require to know the complex number $\mu \equiv \mu_{\tilde{n},+}=\mu_{\tilde{n},-}^{*}$, which for a hexagonal-lattice lead is given by

$$
\mu^{(h)}\left(V_{g}\right)=-\frac{1}{2}\left(V_{g}+i \sqrt{4 \gamma^{2}-V_{g}^{2}}\right),
$$

while for a square-lattice lead

$$
\mu^{(s)}\left(V_{g}\right)=-\frac{1}{2}\left(V_{g}+\gamma+i \sqrt{4 \gamma^{2}-\left(V_{g}+\gamma\right)^{2}}\right) .
$$

Before we describe the general consequences of this observation we first proceed to explore the consequences for the rectangular graphene sample. To make contact to Ref. 8, let us further assume that the strip is very wide, $\mathcal{W} \gg \mathcal{L} \gg a$. The conductance can then be calculated in a saddle-point approximation, which is described in Appendix B. For hexagonal-lattice leads this gives

$$
G=\frac{4 e^{2}}{\pi h} \frac{\mathcal{W}}{\mathcal{L}} \frac{\sqrt{4 \gamma^{2}-V_{g}^{2}}}{V_{g}} \arcsin \frac{V_{g}}{2 \gamma},
$$

which for $V_{g} \rightarrow 0$ recovers the result $G=\frac{4 e^{2}}{\pi h} \frac{\mathcal{W}}{\mathcal{L}}$ derived from the Dirac equation. $\underline{\underline{8}}$ For square-lattice leads one 
finds

$$
G=\frac{4 e^{2}}{\pi h} \frac{\mathcal{W}}{\mathcal{L}} \frac{\sqrt{4 \gamma^{2}-\left(V_{g}+\gamma\right)^{2}}}{V_{g}+\gamma} \arcsin \frac{V_{g}+\gamma}{2 \gamma} .
$$

A similar calculation yields the Fano factor

$$
F=\frac{2 \gamma^{2}}{V_{g}^{2}}-\frac{\sqrt{4 \gamma^{2}-V_{g}^{2}}}{2 V_{g} \arcsin \left(V_{g} / 2 \gamma\right)}
$$

for the hexagonal-lattice leads, and

$$
F=\frac{2 \gamma^{2}}{\left(V_{g}+\gamma\right)^{2}}-\frac{\sqrt{4 \gamma^{2}-\left(V_{g}+\gamma\right)^{2}}}{2\left(V_{g}+\gamma\right) \arcsin \left[\left(V_{g}+\gamma\right) / 2 \gamma\right]}(15)
$$

for the square-lattice leads. For a numerical validation of these results see Section $\nabla$

Equations (12]14) for the hexagonal-lattice leads and (13[15) for the square-lattice leads coincide when the gate potential is shifted by $\gamma$. In particular, for the squarelattice leads the values $G=\frac{4 e^{2}}{\pi h} \frac{\mathcal{W}}{\mathcal{L}}$ of the conductance and $F=1 / 3$ for the Fano factor are now recovered for $V_{g} \rightarrow-\gamma$. This is a direct consequence of the relation

$$
\mu^{(s)}\left(V_{g}\right)=\mu^{(h)}\left(V_{g}+\gamma\right)
$$

between the characteristic self energies $\mu$, Eqs. (10) and (11), which describe the coupling of the leads to the conical points. This relation does not hold for the self energies (9) away from the conical point, but this is not detected in the transport as long as the leads support sufficiently many propagating modes around $n=\tilde{n}$. Surprisingly, Eq. (16) entails that the point of half-filling of the hexagonal-lattice leads (the charge-neutrality point $V_{g}=0$ ) corresponds to the point of three-quarter filling in the square-lattice leads.

\section{EFFECTIVE CONTACT MODEL}

In order to elucidate the generality of this duality between hexagonal- and square-lattice leads, we now liberate ourselves from the strip geometry of the graphene sample, hence, consider samples of more arbitrary geometry in which different transverse modes can be mixed by the transport. In this case the matrix of transmission amplitudes $t_{n^{\prime} n}$ is no longer diagonal, and in general can be obtained from the Fisher-Lee relation 16

$$
S=-1+i \sqrt{v} \Psi^{\dagger} \mathcal{P}^{\dagger}(E-H-\Sigma)^{-1} \mathcal{P} \Psi \sqrt{v} .
$$

The leads are represented by their self energy $\Sigma=$ $\mathcal{P} \Psi \operatorname{diag}\left(\mu_{n,+}\right) \Psi^{\dagger} \mathcal{P}^{\dagger}$, where $\mathcal{P}$ is a coupling matrix of the leads to the contact region. The diagonal matrix $v=\operatorname{diag}\left(-2 \operatorname{Im} \mu_{n,+}\right)$ contains factors proportional to the propagation velocity of the modes in the leads. The matrix $\Psi$ now accounts for the transverse modes in all leads.
According to Eq. (9), the modes in the hexagonal- and square-lattice leads in general have different propagation velocities and self-energies. As a consequence, when these leads are connected to an arbitrary system, described by the internal Hamiltonian $H$, the resulting transport properties in general will differ. When $H$ represents a sufficiently large, weakly doped graphitic system, however, the localization of the Fermi surface near the conical points guarantees that only the lead modes coupling to this part of the Brillouin zone will contribute significantly to the transport. This also applies to other types of leads, different from the two specific cases considered so far, and then yields the universality among contact models advertised in the introduction.

Assuming that the leads support a large number of propagating modes whose properties depend smoothly on the quasi-continuous mode index $n$, we hence can equip all modes in the lead with the same constant $\mu_{n,+} \rightarrow \mu \equiv \mu_{\tilde{n},+}$ characteristic of the modes coupling to the conical points. Using that the transverse mode profiles form an orthogonal set, $\Psi \Psi^{\dagger}=1$, the self energy then simplifies to $\Sigma=\mu \mathcal{P P}^{\dagger}$. For optimally matched leads, $\mathcal{P}=P$ is just a projector of the internal system space onto the contact region. A unitary transformation $S \rightarrow \Psi S \Psi^{\dagger}$ (which does not affect the conductance and the Fano factor) then results in a simplified Fisher-Lee relation for the scattering matrix,

$$
S=-1-(2 i \operatorname{Im} \mu) P^{T}\left(E-H-\mu P P^{T}\right)^{-1} P .
$$

This form of the scattering matrix defines an effective contact model, which is parameterized by a single complex number $\mu$.

For hexagonal-lattice or square-lattice leads, $\mu$ is given by Eq. (10) or Eq. (11), respectively. However, our argumentation entails that the simple effective model Eq. (18) can be applied to a much larger class of leads, which share the same mode-selection principle when coupled to graphene. (An example would be a square lattice with a reduced lattice constant $\sqrt{3} a / q$, where $q$ is an integer.) Within this class of leads, $\mu$ is still given by the self energy $\mu_{\tilde{n},+}$. Depending on the contacts, the matrix $\mathcal{P P}^{\dagger}$ may not be uniform over the contact region, and also may account for a contact tunnel-barrier resistance. Assuming that the coupling strengths $p_{n}=$ $\left\langle\Psi_{n}^{\dagger} \mathcal{P} \mathcal{P}^{\dagger} \Psi_{n}\right\rangle /\left\langle\Psi_{n}^{\dagger} P P^{T} \Psi_{n}\right\rangle$ (where $\Psi_{n}$ is the column vector associated to the $n$th transverse mode) do not depend strongly on the mode index, the effective contact model (18) still holds with $\mu=p_{\tilde{n}} \mu_{\tilde{n},+} \stackrel{15}{\underline{15}}$ Ideal coupling into the graphitic modes near the conical points is realized for a self energy $\mu=-i \gamma$.

\section{APPLICATIONS}

This section describes some consequences of the effective contact model (18), and compares its predictions to the results of numerical computations. 

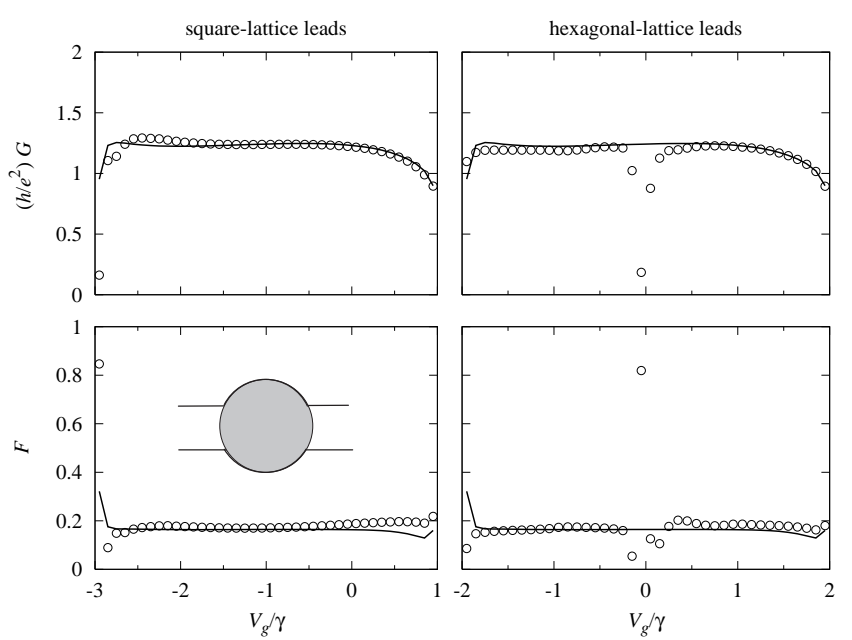

FIG. 3: Gate-voltage dependence of the conductance $G$ (in units of $\left.e^{2} / h\right)$ and the shot-noise Fano factor $F$ for a weakly doped circular graphene sample $\left(E_{F}=\gamma / 10\right)$ of radius $\mathcal{R}=$ $100 \sqrt{3} a$, connected to leads of width $\mathcal{W}=60 \sqrt{3} a$. The data points are obtained by numerical computations for squarelattice leads (left) and hexagonal-lattice leads (right). The curves are the predictions of the effective contact model (18) with $\mu$ given by Eq. (10) or Eq. (11), respectively.

\section{A. Transport in a wide, long graphitic strip and the reconstruction of $\mu$}

Within the effective contact model (18), the conductance of a wide and long graphene strip (as considered in Section (III) is given by

$$
G=\frac{4 e^{2}}{\pi h} \frac{\mathcal{W}}{\mathcal{L}} \frac{\operatorname{Im} \mu}{\operatorname{Re} \mu} \arcsin \frac{\operatorname{Re} \mu}{|\mu|},
$$

while the Fano factor is given by

$$
F=\frac{|\mu|^{2}}{2(\operatorname{Re} \mu)^{2}}-\frac{\operatorname{Im} \mu}{2 \operatorname{Re} \mu \arcsin (\operatorname{Re} \mu /|\mu|)}
$$

(for the derivation see Appendix $\mathrm{B}$ ). These formulae generalize Eqs. (12) - (15), which are recovered when $\mu$ is taken from Eq. (10) (for hexagonal-lattice leads) or Eq. (11) (for square-lattice leads). For other types of leads, the two transport characteristics deliver two independent numbers which can be used to infer the complex number $\mu$.

\section{B. Numerical results}

The validity of the effective contact model can be asserted by numerical computations. The gate-voltage dependence of the conductivity $\sigma$ and the Fano factor $F$ for a sample of width $\mathcal{W}=152 \sqrt{3} a$ and length $\mathcal{L}=30 a$ is shown in Fig. 2. Results of numerical computations obtained by the method of recursive Green's functions ${ }^{17}$ are compared to the theoretical prediction of the effective contact model resulting from Eq. (10) or Eq. (11), as well as to the analytical expressions (12) - (15). Good agreement is found in the range $\left|V_{g}+\gamma\right|<2 \gamma$ for the squarelattice leads, and $\gamma / W \lesssim\left|V_{g}\right|<2 \gamma$ for the hexagonallattice leads, corresponding to the condition that the modes in the vicinity of $\tilde{n}$ are propagating.

As an additional example, Fig. 3 shows the conductance and the Fano factor of a weakly doped circular graphitic region $\left(E_{F}=0.1 \gamma\right)$, calculated for hexagonaland square-lattice leads and within the effective contact model (18), where $\mu$ is again given by Eqs. (10), (11). With the exception of the region $\left|V_{g}\right| \approx \gamma / W$, where the hexagonal-lattice lead does not support many propagating modes, the predictions of the effective contact model again agree nicely with the results for the two types of leads. This shows that the effective contact model remains applicable for graphitic samples where modes are mixed in the transport (so that the transmission matrix is no longer diagonal).

\section{SUMMARY}

This paper critically assesses how sensitively the transport through extended samples of weakly doped graphene depends on the details of the leads and contacts connecting the sample to the electronic reservoirs. The starting point is the observation that there exists a duality between doped graphitic leads and quantum wires, which result in the same transport properties if a gate voltage is suitably adjusted. This duality is not based on similarities of the two type of wires (which would result in very distinct transport properties if attached to a conventional material), but is rooted in a unique mode selection mechanism which originates from the conical points of undoped graphene. Maybe most strikingly, the duality holds even though both types of leads support a different number of propagating modes.

Since the mode selection mechanism is a universal property of weakly doped graphene, the duality described above can be generalized into an effective contact model, which applies to a broad class of leads. The effective model is parameterized by a single complex number $\mu$, which can be determined by a measurement of the conductance and the shot noise of a rectangular undoped graphene strip.

In the present paper, this effective model has been derived using the technical requirement that the leads provide a densely spaced set of propagating modes which couple smoothly to the conical points. This requirement is fulfilled for the typical leads considered in past and present mesoscopic-transport studies, and especially, for ballistic, wide contacts which provide a good electronic coupling to the graphitic sample (so that a transport measurement probes the sample, and not merely the contact resistance).

One potential mechanism to violate the specific tech- 
(a)

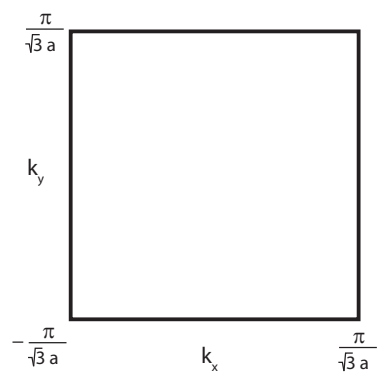

(b)

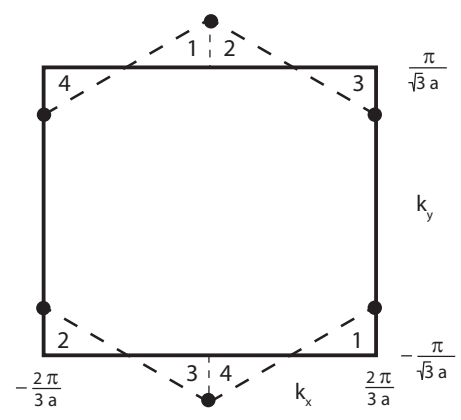

FIG. 4: (a) Brillouin zone for a square lattice with lattice constant $\sqrt{3} a$. (b) Hexagonal Brillouin zone for a honeycomb lattice with lattice constant $a$ (dashed), and its rearrangement into a rectangular Brillouin zones (solid). The numbered triangles denote regions which are transported with reciprocal lattice vectors. The solid dots denote the $\mathrm{K}$ points.

nical assumptions used to derive the effective contact model is strong interface disorder, so that the propagating modes in the leads are mixed by the interface. For an ordinary mesoscopic system, such a diffusive interface changes the specific transport properties decisively (these changes cannot be described by a simple tunnel barrier). For weakly doped graphene, it is conceivable that even such a drastic modification of the contacts does not completely violate the effective contact model. In a transfer description of the contact, the crossing of the diffusive interface from the graphitic sample to the lead results in a translation of the transverse mode profile of the modes close to the conical points into a random superposition of the modes in the lead. It is conceivable that selfaveraging in these random superpositions merely renormalizes the effective self-energy $\mu$ into the average selfenergy of the propagating modes in the lead. This question goes beyond the scope of the techniques used in the present paper, and is left for further consideration.

\section{Acknowledgments}

I gratefully acknowledge helpful discussions with Carlo Beenakker, Edward McCann, and John P. Robinson. This work was supported by the European Commission, Marie Curie Excellence Grant MEXT-CT-2005-023778.

\section{APPENDIX A: MODE MATCHING FOR AN UNDOPED RECTANGULAR GRAPHENE STRIP}

This appendix provides details of the derivation of the transmission eigenvalues (7) for an undoped rectangular graphitic strip coupled to square-lattice or hexagonallattice leads.

A unified description of both cases in real space is facilitated by the discrete coordinate system $(l, m)$, shown in Fig. 1. Analogously, Fig. 4 shows a convenient choice of the Brillouin zones which facilitates a unified description in $k$ space. For the square lattice with lattice constant $\sqrt{3} a$, the Brillouin zone is given by the standard square $\left|k_{x}\right|,\left|k_{y}\right| \leq \frac{\pi}{\sqrt{3} a}$. Using the periodicity of the band structure, the conventional hexagonal Brillouin zone for the honeycomb lattice is rearranged into the rectangle $\left|k_{x}\right| \leq \frac{2 \pi}{3 a} \equiv q_{x},\left|k_{y}\right| \leq \frac{\pi}{\sqrt{3} a} \equiv \frac{3}{2} q_{y}$, where $\left|k_{x}\right|=q_{x}$, $\left|k_{y}\right|=q_{y}$ denotes the position of the conical points. With this choice, both Brillouin zones have the same extent in $k_{y}$ direction. Moreover, the boundary conditions of the tight-binding model select the same transverse wavenumbers $k_{y, n}=\frac{n \pi}{\mathcal{W}+\sqrt{3} a}$. The transverse mode profile $\Psi_{n m}$ is then uniform throughout the system, and given by Eq. (4).

For fixed transverse wavenumber $k_{y, n}$, the longitudinal wavenumbers $k_{n}$ follow from the dispersion relations (5) and (6), respectively. For each transverse wavenumber, there are two solutions of opposite sign.

In the undoped central graphene regions, the longitudinal wavenumbers are complex, and the modes are evanescent with decay constant $\operatorname{Im} k_{n}=\kappa_{n}$ given in Eq. (8). The wave functions $\phi_{A}, \phi_{B}$ on the $A$ and $B$ sites are determined by the Schrödinger equation, which delivers

$$
\begin{aligned}
& \phi_{A}^{(n)}(l, m)=\alpha e^{-3 a l \kappa_{n}+2 \pi i l} \Psi_{n m}, \\
& \phi_{B}^{(n)}(l, m)=\beta e^{3 a l \kappa_{n}+2 \pi i l} \Psi_{n m},
\end{aligned}
$$

respectively, where $\alpha$ and $\beta$ are constants.

The Landauer approach requires to match these wavefunctions to the propagating modes in the lead. These modes are characterized by a real longitudinal wavenumbers $\pm k_{n}$, where for definiteness $k_{n}>0$ is taken to be the solution in the right half of the Brillouin zone.

On the hexagonal lattice, modes with a positive longitudinal wavenumber $k_{n}>0$ are right-propagating when $\operatorname{sgn} V_{g}=\eta>0$, (so that the Fermi energy lies below the conical point), while these modes are left-propagating for $\eta<0$ (since the band structure above the conical point has the opposite curvature). For a particle incident from the left, the solution of the Schrödinger equation in the left lead takes the form

$$
\begin{aligned}
& \phi_{A, L}^{(n)}(l, m)=C \eta\left(f_{n,-\eta k_{n}} / f_{n, \eta k_{n}}\right)^{1 / 4} e^{3 i \eta k_{n} a l} \Psi_{n m} \\
& \quad+C r_{n n} \eta\left(f_{n,-\eta k_{n}} / f_{n,-\eta k_{n}}\right)^{1 / 4} e^{-3 i \eta k_{n} a l} \Psi_{n n}(\mathrm{~A} 2 \mathrm{a}) \\
& \phi_{B, L}^{(n)}(l, m)=C\left(f_{n, \eta k_{n}} / f_{n,-\eta k_{n}}\right)^{1 / 4} e^{3 i \eta k_{n} a l} \Psi_{n m} \\
& \quad+C r_{n n}\left(f_{n,-\eta k_{n}} / f_{n, \eta k_{n}}\right)^{1 / 4} e^{-3 i \eta k_{n} a l} \Psi_{n m},(\mathrm{~A} 2 \mathrm{~b})
\end{aligned}
$$

while in the right lead it is given by

$$
\begin{aligned}
& \phi_{A, L}^{(n)}(l, m)=C t_{n n} \eta\left(f_{n,-\eta k_{n}} / f_{n, \eta k_{n}}\right)^{1 / 4} e^{3 i \eta k_{n} a(l-L)} \Psi_{n m}, \\
& (\mathrm{~A} 2 \mathrm{c}) \\
& \phi_{B, L}^{(n)}(l, m)=C t_{n n}\left(f_{n, \eta k_{n}} / f_{n,-\eta k_{n}}\right)^{1 / 4} e^{3 i \eta k_{n} a(l-L)} \Psi_{n m} .
\end{aligned}
$$

Here $C$ is an arbitrary coefficient, while $r_{n n}$ and $t_{n n}$ are the reflection and transmission coefficient, respectively. 
On the square lattice, positive longitudinal wavenumbers $k_{n}>0$ are always associated with right-propagating modes. The wave function in the left lead can hence be written as

$$
\begin{aligned}
\phi_{L}^{(n)}(l, m)= & C e^{\sqrt{3} i k_{n} a(l+1 / 2)} \Psi_{n m} \\
& +C r_{n n} e^{-\sqrt{3} i k_{n} a(l+1 / 2)} \Psi_{n m}
\end{aligned}
$$

while in the right lead

$$
\phi_{R}^{(n)}(l, m)=C t_{n n} e^{\sqrt{3} i k_{n} a(l-L-1 / 2)} \Psi_{n m} .
$$

Using the fact that the wavefunctions (A1), (A2), (A3), all satisfy the Schrödinger equation when the graphitic strip or the leads would be formally extended beyond the interface, the matching conditions take the simple form of continuity requirements

$$
\begin{aligned}
& \phi_{A}^{(n)}(0, m)=\phi_{A, L}^{(n)}(0, m), \\
& \phi_{B}^{(n)}(0, m)=\phi_{B, L}^{(n)}(0, m), \\
& \phi_{A}^{(n)}(L, m)=\phi_{A, R}^{(n)}(L, m), \\
& \phi_{B}^{(n)}(L, m)=\phi_{B, R}^{(n)}(L, m)
\end{aligned}
$$

for the hexagonal-lattice leads, and

$$
\begin{aligned}
& \phi_{A}^{(n)}(0, m)=\phi_{L}^{(n)}(-1, m), \\
& \phi_{B}^{(n)}(0, m)=\phi_{L}^{(n)}(0, m), \\
& \phi_{A}^{(n)}(L, m)=\phi_{R}^{(n)}(L, m), \\
& \phi_{B}^{(n)}(L, m)=\phi_{R}^{(n)}(L+1, m)
\end{aligned}
$$

for the square-lattice leads.

The resulting linear systems of equations deliver the transmission coefficient $t_{n n}$ in the form (7), where for each lattice the self energy is defined in Eq. (9).

\section{APPENDIX B: CONDUCTANCE AND FANO FACTOR FOR WIDE AND LONG GRAPHENE STRIPS}

This appendix describes the saddle-point approximation which is used to obtain the conductance (19) and the Fano factor (20) of an undoped graphene strip of width $\mathcal{W} \gg \mathcal{L} \gg a$. The conductance (12), (13) and the Fano factor (14), (15) for hexagonal-lattice and square-lattice leads follow when these expressions are evaluated with (10) and (11), respectively.

Let us start with a detailed account for the conductance, which in general is related to the transmission coefficients $t_{n m}$ via the Landauer formula (2). The transmission matrix (17) of the graphene strip is diagonal, hence $G=\left(2 e^{2} / h\right) \sum_{n}\left|t_{n n}\right|^{2}$. Since the strip is wide, $\mathcal{W} \gg \mathcal{L} \gg a$, the transverse wavenumbers are closely spaced and the transmission coefficients (7) depend quasi-continuously on the mode index $n$, so that the sum can be replaced by an integral,

$$
G=\frac{2 e^{2}}{h} \int\left|\frac{\mu_{n,+}-\mu_{n,-}}{\mu_{n,+} e^{-\kappa_{n} \mathcal{L}}-\mu_{n,-} e^{\kappa_{n} \mathcal{L}}}\right|^{2} d n .
$$

For $\mathcal{L} \gg a$, all transmission coefficients decay rapidly with the exception of modes with index $n \approx 2(W+1) / 3 \equiv$ $\tilde{n}$, which have transverse wave numbers in the vicinity of the conical points and hence a small decay coefficient $\kappa_{n}$, given in Eq. (8). Hence the integrand in Eq. (B1) has a pronounced maximum at $n \approx \tilde{n}$. Away from this maximum, for $n=\tilde{n}+\delta$, the integrand decays rapidly because of the finite decay constant $\kappa_{n} \approx \delta \pi / \mathcal{W}$, and attains very small values on a scale on which the selfenergies $\mu_{n,+}=\mu_{n,-}^{*}$ barely change. One hence can approximate the self energy by the value $\mu_{n,+} \approx \mu_{\tilde{n},+} \equiv \mu$ at the conical point. Furthermore, the integration limits can be extended to $\pm \infty$. The resulting integral

$$
G=\frac{2 e^{2}}{h} \int_{-\infty}^{\infty} \frac{2(\operatorname{Im} \mu)^{2}}{|\mu|^{2} \cosh (2 \delta \pi \mathcal{L} / \mathcal{W})-(\operatorname{Re} \mu)^{2}+(\operatorname{Im} \mu)^{2}} d \delta
$$

can be calculated exactly, and results in Eq. (19).

For the Fano factor (3) one is led to calculate a similar integral $I \equiv \int\left|t_{n n}\right|^{4} d n$, which can be evaluated using exactly the same steps as for the conductance. The integral is then approximated as

$$
I=\int_{-\infty}^{\infty} \frac{4(\operatorname{Im} \mu)^{4}}{\left[|\mu|^{2} \cosh (2 \delta \pi \mathcal{L} / \mathcal{W})-(\operatorname{Re} \mu)^{2}+(\operatorname{Im} \mu)^{2}\right]^{2}} d \delta
$$

which again can be evaluated exactly,

$$
I=\frac{\mathcal{W}}{\pi \mathcal{L}} \frac{(\operatorname{Im} \mu)^{2}}{(\operatorname{Re} \mu)^{2}}-\frac{\mathcal{W}}{\pi \mathcal{L}} \frac{\operatorname{Im} \mu\left[(\operatorname{Im} \mu)^{2}-(\operatorname{Re} \mu)^{2}\right]}{(\operatorname{Re} \mu)^{3}} \arcsin \frac{\operatorname{Re} \mu}{|\mu|} .
$$

The final result for the Fano factor is given in Eq. (20).
${ }^{1}$ K. S. Novoselov, A. K. Geim, S. V. Morozov, D. Jiang, Y. Zhang, S. V. Dubonos, I. V. Grigorieva, and A. A. Firsov Science 306, 666 (2004).

${ }^{2}$ K. S. Novoselov, A. K. Geim, S. V. Morozov, D. Jiang, Y. Zhang, S. V. Dubonos, I. V. Grigorieva, and A. A. Firsov, Nature 438, 197 (2005).

3 Yuanbo Zhang, Yan-Wen Tan, Horst L. Stormer, and
Philip Kim, Nature 438, 201 (2005).

${ }^{4}$ Y. Zhang, Z. Jiang, J. P. Small, M. S. Purewal, Y.-W. Tan, M. Fazlollahi, J. D. Chudow, J. A. Jaszczak, H. L. Stormer, and P. Kim, Phys. Rev. Lett. 96, 136806 (2006).

5 D. P. DiVincenzo and E. J. Mele, Phys. Rev. B 29, 1685 (1984); T. Ando, T. Nakanishi, and R. Saito, J. Phys. Soc. Japan 67, 2857 (1998); Y. Zheng and T. Ando, Phys. Rev. 
B 65, 245420 (2002); V. P. Gusynin and S. G. Sharapov, Phys. Rev. Lett. 95, 146801 (2005).

6 E. McCann, K. Kechedzhi, Vladimir I. Fal'ko, H. Suzuura, T. Ando, and B. L. Altshuler, Phys. Rev. Lett. 97, 146805 (2006).

7 S. V. Morozov, K. S. Novoselov, M. I. Katsnelson, F. Schedin, L. A. Ponomarenko, D. Jiang, and A. K. Geim, Phys. Rev. Lett. 97, 016801 (2006).

8 J. Tworzydło, B. Trauzettel, M. Titov, A. Rycerz, and C. W. J. Beenakker, Phys. Rev. Lett. 96, 246802 (2006); I. Snyman and C. W. J. Beenakker, Phys. Rev. B 75, 045322 (2007); A. R. Akhmerov and C. W. J. Beenakker, Phys. Rev. B 75, 045426 (2007).

9 N. M. R. Peres, F. Guinea, and A. H. Castro Neto, Phys. Rev. B 73, 125411 (2006); J. A. Vergés, F. Guinea, G. Chiappe, and E. Louis, Phys. Rev. B 75, 085440 (2007).

10 I. L. Aleiner and K. B. Efetov, Phys. Rev. Lett. 97, 236801 (2006); A. Altland, Phys. Rev. Lett. 97, 236802 (2006); P. M. Ostrovsky, I. V. Gornyi, and A. D. Mirlin, Phys. Rev.
B 74, 235443 (2006).

11 V. V. Cheianov and V. I. Fal'ko, Phys. Rev. B 74, 041403(R) (2006).

12 E. McCann, Phys. Rev. B 74, 161403(R) (2006).

13 C. W. J. Beenakker, Rev. Mod. Phys. 69, 731 (1997).

14 Y. M. Blanter and M. Büttiker, Phys. Rep. 336, 1 (2000).

15 The contact model can be extended to accommodate the case of leads with different coupling to each of the conical points $\mathrm{K}, \mathrm{K}^{\prime}$, each characterized by a different complex number $\mu_{K}, \mu_{K^{\prime}}$. The self energy has then to be decomposed into two parts. Similar decompositions are required when the graphitic region is coupled to several inequivalent leads $i$ (e.g., graphene strips of different doping), or if the Fermi surface of the lead around $\tilde{n}$ is nested.

16 D. S. Fisher and P. A. Lee, Phys. Rev. B 23, 6851 (1981).

17 H. U. Baranger, D. P. DiVincenzo, R. A. Jalabert, and A. D. Stone, Phys. Rev. B 44, 10637 (1991). 